\title{
A Novel Compound Control Method for Hydraulically Driven Shearer Drum Lifting
}

\author{
Lei Si, ${ }^{1}$ Zhongbin Wang, ${ }^{1}$ Xinhua Liu, ${ }^{1,2}$ and Lin Zhang ${ }^{1}$ \\ ${ }^{1}$ School of Mechatronic Engineering, China University of Mining \& Technology, Xuzhou 221116, China \\ ${ }^{2}$ Xuyi Mine Equipment and Materials R\&D Center, China University of Mining \& Technology, Huaian 211700, China \\ Correspondence should be addressed to Zhongbin Wang; wangzbpaper@126.com
}

Received 21 August 2014; Accepted 29 October 2014; Published 20 November 2014

Academic Editor: Kalyana C. Veluvolu

Copyright (c) 2014 Lei Si et al. This is an open access article distributed under the Creative Commons Attribution License, which permits unrestricted use, distribution, and reproduction in any medium, provided the original work is properly cited.

\begin{abstract}
In order to adjust shearer drum swiftly and precisely to adapt to the changes of coal seam, a compound control approach based on cerebellar model articulation control and fractional order PID controller was proposed. As the movement precision and response speed of hydraulic system were determined mainly by the control precision of valve-controlled asymmetrical hydraulic cylinder, its working principle and characteristics were analyzed in this paper, with particular focusing on the asymmetry problem. Furthermore, RBF neural network was applied to obtaining reasonable tuning parameters and a control algorithm of proposed controller was designed. Finally, laboratory experiments were developed to verify the validity and effectiveness of proposed compound control method. The testing results, compared with those for other controllers, proved that the proposed compound control method can acquire high movement precision and respond speed in the system of hydraulically driven shearer drum lifting with different control conditions.
\end{abstract}

\section{Introduction}

With the development of coal mining technology and stringent requirement for colliery safety, the automation control of fully mechanized coal face has become an inexorable trend. As the core mining equipment, shearer plays a decisive function in achieving safe and high-efficiency exploitation, and its automation control is always an interested research subject in coal mining field [1]. With years of development, many researchers have proposed the technology of memory cutting to achieve the automation control of shearer [2-5]. However, the control effect of memory cutting depends on the performance of hydraulic adjustment height system of shearer drum. As one of the most important constituent parts in shearer automation control system, its performance directly affects the efficiency and reliability of coal mining. It is noteworthy that hydraulically driven shearer drum lifting has become one of the key technologies to improve the performance of hydraulic system [6].

The hydraulically driven shearer drum lifting is that when the shearer is working, the drum height is automatically adjusted along the coal-rock interface of coal face to avoid cutting rock. It can improve the quality of coal production and extend the service life of shearer. The lifting of drum height is achieved through the displacement of hydraulic cylinder piston. Due to different mining geological conditions, the coal seam thickness between roof and floor has a great discrepancy. Therefore, the hydraulic cylinder is needed to carry on a fast response to the control signal, so as to avert cutting the roof and floor.

Figure 1 shows a model of hydraulically driven shearer drum lifting developed by the China University of Mining and Technology. The shearer drum lifting is realized through a valve-controlled asymmetrical hydraulic cylinder (VCAHC) and the valve is an electrohydraulic proportional directional valve in this system. An angle sensor is installed on the swing-arm to measure the output of the VCAHC and the deviation of VCAHC output, and the control signal is processed through a controller as the input of electrohydraulic valve.

In the system of hydraulically driven shearer drum lifting, hydraulic cylinder is a crucial component converting the fluid power into linear motion and achieving the adjustment of drum height. Recently, hydraulic cylinders are used 


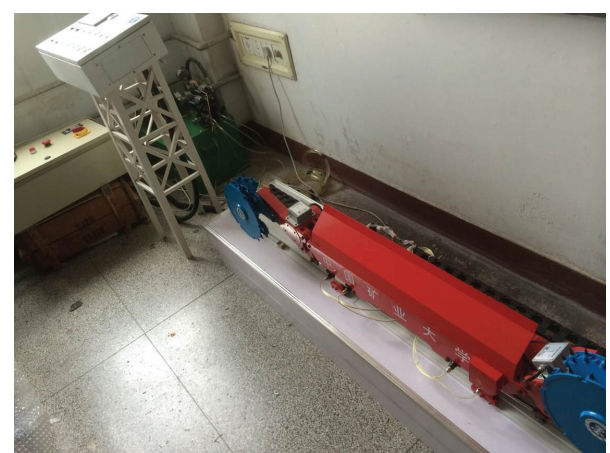

FIGURE 1: Hydraulically driven shearer drum lifting developed by the China University of Mining and Technology.

widespread with the proportional valves, which are electronically controlled. The important required functions of the above hydraulic system are accurate position control and fast response. In order to achieve these functions, it is necessary to apply convenient control strategies. In industrial applications, conventional control methods such as PD and PID are commonly used. However, traditional control methods have linear characteristics and imperfect control effect. Therefore, they are insufficient to overcome nonlinearities which exist in the nature of this hydraulic system $[7,8]$.

Cerebellar model articulation control (CMAC) is a new type of neural network first advanced by Almeida and Simões [9]. It is a local-learning-based control, which is very suitable for real-time control of the electrohydraulic proportional control system, with its simple network structure, high convergence precision, and rapid learning speed [10]. CMAC has been applied in many research fields, including function approach, dynamic modeling, and pattern recognition [1113] and has provided a new solution to the problem of valvecontrolled hydraulic cylinder control design as well.

In order to solve the control problems of hydraulically driven shearer drum lifting, this paper proposed a new compound control method based on CMAC and fractional order PID (CMAC-PI ${ }^{\lambda} \mathrm{D}^{\mu}$ ) controller to control the hydraulic system. The rest of the paper was organized as follows. Some related works were outlined based on literature in Section 2. The working principle and mathematical model of the system were presented in Section 3. A novel compound control method and CMAC-PI ${ }^{\lambda} \mathrm{D}^{\mu}$ controller were derived and designed in Section 4. Some laboratory experiments were put forward to validate the control effect of proposed method in Section 5. Finally, our conclusions were summarized in Section 6.

\section{Literature Review}

Recent publications relevant to this paper are mainly concerned with the research stream of the control principles and control methods for hydraulically driven shearer drum lifting. In this section, we try to summarize the relevant literatures.
Currently, many scholars have worked on the system of hydraulically driven shearer drum lifting. Liu et al. applied fuzzy theory and grey correlation calculation in analyzing automatic control of shearer drum according to the switch control characteristic of valve-controlled hydraulic cylinder $[2,3]$. Wang and Song analyzed the nonlinear characteristics of hydraulic height adjustment system and presented an adaptive control strategy based on BP algorithm and fuzzy neural network [14]. Quan et al. provided a fuzzy adaptive PID controller to simulate and analyze the real time and stability of hydraulic height adjustment system [15]. In [4], the memory cutting technology and artificial immune algorithm were adopted in hydraulic height adjustment system to realize the self-adaptive adjustment height of shearer drum. Fan et al. proposed a grey-Markovian model in the hydraulic system to overcome the defects of traditional shearer memory cutting strategy in adjusting rocker arm height frequently and manually [5]. In [16], a height-adjustment cylinder model was established to deduce the state space equation of the shearer height adjusting system and the sliding-mode variable structure control strategy was adopted as an automatic height adjusting controller.

For the hydraulically driven shearer drum lifting, the control methods of valve-controlled hydraulic cylinder mainly include self-adaptive control, robust control, and slidingmode control. For instance, [17] had designed a kind of robust controller based on the PD control structure, which could output different nonlinear compensations according to specific working conditions. Reference [18] designed a nonlinear controller based on backstepping approach for controlling force of a single rod hydraulic actuator and a PI observer was used to estimate the states of the system. References $[19,20]$ described the development of an adaptive fuzzy sliding mode controller for an electrohydraulic system with unknown dead zone. Reference [21] investigated a robust velocity control problem for a hydraulic elevator including cylinder friction, pump friction, and pump leakage. In [22], an iterative learning control (ILC) method was applied to tracking the displacement curve of the hydraulic press slider and a fuzzy ILC algorithm utilized the fuzzy strategy to adaptively adjust the iterative learning gains. In [23], a new sliding mode control with varying boundary layers was proposed to improve the tracking performance of a nonlinear electrohydraulic position servosystem. Reference [24] presented the development of a proportional-integralderivative- (PID-) based control method for application to active vehicle suspension systems (AVSS). In [25], a method of control strategy was implemented by employing a fuzzy logic controller (FLC) whose parameters were optimized using particle swarm optimization (PSO). In [26], the position control of asymmetrical hydraulic cylinder with two chambers connected was carried out by the use of single, two-position, and two-way proportional valve, and a sliding mode controller was applied in analyzing the stability of the control system. In [27], an optimally tuned nonlinear PID controller for hydraulic systems was designed and an estimated process model was used for tuning optimal PID parameters in the case of variable process parameters. In [28], a fuzzy controller system, consisting of one fuzzy 
coordination controller and one fuzzy tracking controller, was proposed to achieve a synchronous positioning objective for a dual-cylinder electrohydraulic lifting system with unbalanced loading, system uncertainties, and disturbances. In [29], a compound controller incorporating both PID control and wavelet network control was presented for online, realtime control by dynamics compensation. In [30], a new CMAC-based control method was presented to improve the precision of the valve-controlled asymmetrical cylinder and the hydraulically driven six degrees of freedom parallel manipulator movement.

When focused on the literatures mentioned above, it can be deduced that intelligent and robust nonlinear controllers are necessity for hydraulic system in order to obtain accurate and precise control performance. However, the performance and control effects of the hydraulically driven shearer drum lifting under different working conditions were not analyzed, and the shearer drum could not be adjusted swiftly and precisely to adapt to the changes of coal seam. Therefore, a new controller named as CMAC-PI ${ }^{\lambda} \mathrm{D}^{\mu}$ controller was applied for position control of the hydraulically driven shearer drum lifting.

\section{Mathematical Model of VCAHC}

In the hydraulic system, the asymmetrical cylinder with a single pole is adopted to lift the drum. Compared with the symmetrical cylinder with two poles, the asymmetrical cylinder has many advantages in industrial applications, including a compact working space, a simple structure, ease of production, and reliably oil-proof feature [31]. However, the working characteristics of VCAHC are much different from those of the valve-controlled symmetrical hydraulic cylinder, due to the structural dissimilarity. Figure 2 illustrates the VCAHC structure, where input $e$ is the drive voltage of the electrohydraulic valve, $x_{v}$ is the displacement of the valve core, $x_{p}$ is the displacement of the piston, $p_{s}$ is the oilsupply pressure, $p_{o}$ is pressure of the oil return;, $p_{1}$ and $p_{2}$ are the respective pressures in the two cavities of the cylinder, $A_{1}$ and $A_{2}$ are the piston areas of the two sides $\left(\tau=A_{2} / A_{1}<1\right)$, respectively, $M$ is the equivalent mass of the piston and load, and $F_{L}$ is the external load.

In order to establish the mathematical model of VCAHC, the loading flow characteristics of VCAHC should be analyzed and some suitable conditions are assumed as: the orifices of the electrohydraulic valve are matching and symmetrical; the oil-supply pressure is constant and pressure of the oil return is zero nearly; the elastic and damped loading is ignored. The mathematical model of $x_{p}$ and $x_{v}, F_{L}$ can be derived according to some simplified representations and Laplace transform, which can be expressed as follows:

$$
X_{p}(s)=\frac{\left(K_{q} / A_{1}\right) X_{V}(s)-\left(K_{\mathrm{ce}} / A_{1}^{2}+\left(V_{t} / 4 \beta_{e} A_{1}^{2}\right) s\right) F_{L}(s)}{s\left(\left(1 / \omega_{h}^{2}\right) s^{2}+\left(2 \zeta_{h} / \omega_{h}\right) s+1\right)},
$$

where $K_{q}$ is the flow gain of the valve in the zero position, $K_{\text {ce }}$ is the flow-pressure coefficient, $V_{t}$ is the equivalent volume,

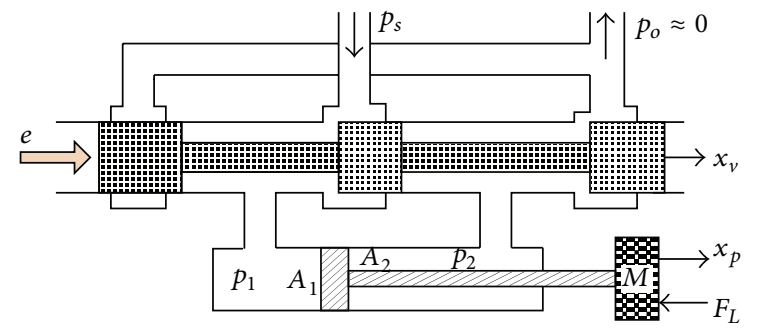

FIGURE 2: Structure of the valve-controlled asymmetrical hydraulic cylinder.

$\beta_{e}$ is the effective modulus of volume elasticity, $\omega_{h}$ is the natural frequency of cylinder, and $\zeta_{h}$ is the hydraulic damping coefficient.

As the structural asymmetry of hydraulic cylinder, the characteristics of positive movement and negative movement are significantly distinct, which result in differentiating the values of $K_{q}, K_{\mathrm{ce}}, V_{t}$, and $\zeta_{h}$. Among these parameters, the variation of $K_{q}$ and $V_{t}$ has a significant influence on the dynamic performance of VCAHC. Equations (2) define $K_{q}$ and $V_{t}$ for both positive movement and negative movement, where $C_{d}$ and $\varsigma$ are the throttle coefficient and valve area gradient, respectively, $\rho$ is the oil density, $L$ is the piston stroke, and $p_{L}\left(p_{L}=p_{1}-\tau p_{2}\right)$ is the loading pressure:

$$
\begin{gathered}
K_{q}= \begin{cases}K_{q}^{+}=C_{d} \varsigma \sqrt{\frac{2\left(p_{s}-p_{L}\right)}{\left(\rho\left(1+\tau^{3}\right)\right)}} \quad \dot{x}_{p}>0, \\
K_{q}^{-}=C_{d} \varsigma \sqrt{\frac{2 \tau^{3}\left(p_{s}-p_{L}\right)}{\left(\rho\left(1+\tau^{3}\right)\right)}} \quad \dot{x}_{p}<0,\end{cases} \\
V_{t}= \begin{cases}V_{t}^{+}=\frac{2 L A_{1}}{\left(1+\tau^{3}\right)} & \dot{x}_{p}>0, \\
V_{t}^{-}=\frac{2 \tau^{3} L A_{2}}{\left(1+\tau^{3}\right)} & \dot{x}_{p}<0 .\end{cases}
\end{gathered}
$$

The degree of asymmetry $\bar{\omega}$ is the degree of difference between positive movement and negative movement. The value of $\bar{\omega}$ is very difficult to be obtained by a precise expression and is determined mainly by $\tau$ and $p_{L}$, among others. When VCAHC is applied in the hydraulic system, the following equation can be used to calculate $\bar{\omega}$ based on the position error expressed as

$$
\bar{\omega}=\frac{2\left(e^{+}-e^{-}\right)}{e^{+}+e^{-}} \times 100 \%,
$$

where $e^{+}$and $e^{-}$represent the positive and negative position errors, respectively.

The electrohydraulic proportional valve is composed of electrical part and hydraulic part. The displacement of the valve core is generated through the electrical part and the kinematics equation can be expressed as follows:

$$
\frac{\mathrm{d}^{2} x_{v}}{\mathrm{~d} t}+2 \delta_{v} \omega_{n} \frac{\mathrm{d} x_{v}}{\mathrm{~d} t}+\omega_{n}^{2} x_{v}=K_{a} K_{v} \omega_{n}^{2} e,
$$


where $\omega_{n}$ and $\delta_{v}$ are the $-3 \mathrm{~dB}$ bandwidth and damping ratio of the valve, respectively, $K_{a}$ is the amplifier gain, and $K_{v}$ is the flow gain of valve.

After the Laplace transform, the transfer function of the valve can be expressed as follows:

$$
\frac{X_{V}(s)}{E(s)}=\frac{K_{a} K_{v}}{s^{2} / \omega_{n}^{2}+2 \delta_{v} s / \omega_{n}+1} .
$$

Equations (1) and (4) can show that the denominator of the open-loop transfer function of the system is five orders and is very complex to be analyzed. In general, compared with hydraulic cylinder, the valve has more fast response speed. Therefore, the valve has smaller influence on the response characteristics of the cylinder and can be regarded as a proportion cycle.

The asymmetrical movement of VCAHC caused by its asymmetrical structure has a great influence on control precision; indeed, it is very difficult to achieve ideal control with traditional PID controller. However, with high flexibility of fractional order PID, a compound controller based on CMAC neural network and fractional order PID can provide a feasible solution for control of severely asymmetrical VCAHC.

\section{Design of CMAC and Fractional Order PID Controller}

4.1. CMAC Neural Network. The CMAC neural network is a kind of neural networks that imitate the structure of human cerebellum. It is a useful technique which is a table lookup neuron computing technique, faster learning convergence property, and good generalization ability. In a CMAC neural network, associated memory is activated by the input signals triggering similar memory cells. A weighting value is saved in the memory address. When a signal is input, a memory set is activated after the memory addresses are quantified, coded, and combined. An output that corresponds to the input signal is produced after the weighting values in the activated memory addresses are summed. Furthermore, the difference between distorted and ideal output signals can be used to adjust the weighting values of the activated memory addresses, generating a more realistic output from the reinputted distorted signals.

The mathematical principle and structure of CMAC can be described by the $S \rightarrow M \rightarrow W \rightarrow Y$ serial mapping displayed by Figure 3, where $S$ is the combined total input vectors, including $S_{1}, S_{2}, \ldots, S_{n}, M$ represents the space of concept storage, $W$ is the space of actual storage, and $Y$ is the network output.

The principle of CMAC can be described by a series of working procedures. Every vector in state space $S$, as an address variable, is mapped into concept storage $M$ by a combination method, which can be realized through an associative relationship between $S$ and $M$. The relationship can be measured by the number of associative elements expressed as $c$. With growth in the number of input vectors, the size of concept storage $M$ will become huge. Therefore, pseudorandom mapping methods, such as Hash encoding,

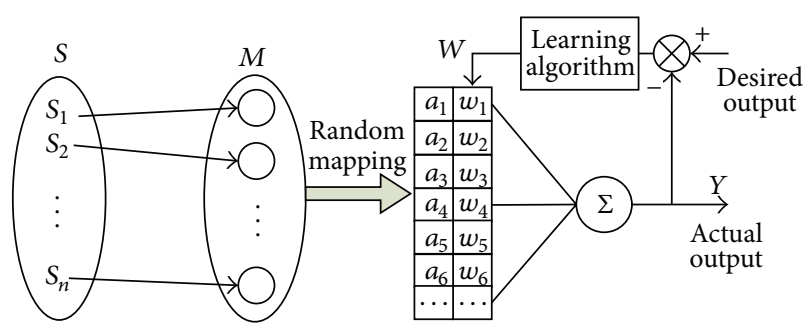

Figure 3: Principle and structure of CMAC.

are often employed to reduce the size of storage space. This method can map a huge storage $M$ into $c$ smaller storages in $W$. The final output of CMAC is generated by the sum of weights owned by $c$ elements, and the difference between actual output and desired output is used to update the weights stored in $W$. The learning process is often accelerated through gradient descent calculation [32].

4.2. Fractional Order PID Controller. Fractional order PID $\left(\mathrm{PI}^{\lambda} \mathrm{D}^{\mu}\right)$ controller is the generalized expression form of integer order PID controller. The orders of integration term $\lambda$ and differential term $\mu$ are extended to fractional field, which both can take any value of complex numbers, and the transfer function of $\mathrm{PI}^{\lambda} \mathrm{D}^{\mu}$ controller can be described as follows:

$$
G_{c}(s)=K_{p}+K_{i} s^{-\lambda}+K_{d} s^{\mu} \quad \lambda, \mu \in \mathbf{R} .
$$

Observed from (6), the traditional integer order PID controller is a special case of $\lambda=\mu=1$. For $\lambda=\mu=0$, the $P$ controller is obtained, and for $\lambda=0, \mu=1$ the PD controller is obtained, and when $\lambda=1, \mu=0$, the PI controller is obtained.

In order to program the fractional order PID controller, the discretization form of its transfer function can be described as follows:

$$
\begin{aligned}
u_{\mathrm{PID}}(k)= & K_{p} \operatorname{error}(k)+K_{i} T^{\lambda} \sum_{i=0}^{k} q_{i} \operatorname{error}(k-i) \\
& +K_{d} T^{-\mu} \sum_{i=0}^{k} d_{i} \operatorname{error}(k-i),
\end{aligned}
$$

where $T$ is the sampling time, $k$ is the sampling times, $q_{i}=$ $[1-(1+\lambda) / i] q_{i-1}, q_{0}=1$, and $d_{i}=[1-(1-\mu) / i] d_{i-1}, d_{0}=1$.

Obviously, $\mathrm{PI}^{\lambda} \mathrm{D}^{\mu}$ controller mainly has five parameters, including $K_{p}, K_{i}, K_{d}, \lambda$, and $\mu$. In order to improve the control effect preferably and accurately, RBF neural network is adopted to obtain reasonable tuning parameters. The structure and principle of RBF network tuning $\mathrm{PI}^{\lambda} \mathrm{D}^{\mu}$ controller are shown in Figure 4.

$\mathrm{RBF}$ is a kind of three-layer feedforward network. The mapping from input to output is nonlinear and the mapping from hidden layer to output layer is linear, which greatly accelerates the learning speed and avoids local minimum problem. In the structure of RBF network, the input vector is $\mathbf{X}=\left[x_{1}, x_{2}, \ldots, x_{l}\right]^{\mathrm{T}}$ and the radial basis vector 


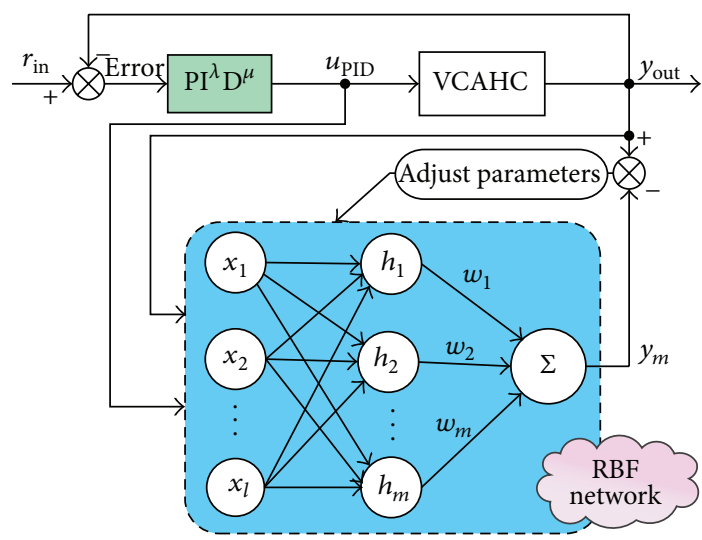

FIGURE 4: The structure and principle of RBF network tuning $\mathrm{PI}^{\lambda} \mathrm{D}^{\mu}$ controller.

is $\mathbf{H}=\left[h_{1}, h_{2}, \ldots, h_{m}\right]^{\mathrm{T}}$, where $h_{j}$ is the Gauss function, expressed as follows:

$$
h_{j}=\exp \left[-\frac{\left\|\mathbf{X}-\mathbf{C}_{j}\right\|^{2}}{\left(2 b_{j}^{2}\right)}\right], \quad j=1,2, \ldots, m,
$$

where $m$ is the number of nodes in hidden layer, $\mathbf{C}_{j}$ is connection vector of the $j$ th node, $\mathbf{C}_{j}=\left[c_{j 1}, c_{j 2}, \ldots, c_{j l}\right]^{\mathrm{T}}$, and $b_{j}$ is the basis width of the $j$ th node and $b_{j}>0$.

The weight vector of the network is marked as $\boldsymbol{\omega}=$ $\left[\omega_{1}, \omega_{2}, \ldots, \omega_{m}\right]^{\mathrm{T}}$. The output of network can be calculated as follows:

$$
y_{m}(k)=\boldsymbol{\omega}^{\mathrm{T}} \mathbf{H}
$$

According to the gradient descent algorithm, the corresponding parameters of RBF network can be updated through the following equations:

$$
\begin{aligned}
& \omega_{j}(k)=\omega_{j}(k-1)+\eta\left[y_{\text {out }}(k)-y_{m}(k)\right] h_{j} \\
& +\alpha\left[\omega_{j}(k-1)-\omega_{j}(k-2)\right], \\
& \Delta b_{j}=\left[y_{\text {out }}(k)-y_{m}(k)\right] h_{j} \omega_{j} \frac{\left\|\mathbf{X}-\mathbf{C}_{j}\right\|^{2}}{b_{j}^{3}}, \\
& b_{j}(k)=b_{j}(k-1)+\eta \Delta b_{j} \\
& +\alpha\left[b_{j}(k-1)-b_{j}(k-2)\right], \\
& \Delta c_{j i}=\left[y_{\text {out }}(k)-y_{m}(k)\right] \omega_{j} \frac{\left(x_{j}-c_{j i}\right)}{b_{j}^{2}}, \\
& c_{j i}(k)=c_{j i}(k-1)+\eta \Delta c_{j i} \\
& +\alpha\left[c_{j i}(k-1)-c_{j i}(k-2)\right],
\end{aligned}
$$

where $\eta$ is the learning rate and $\alpha$ is the momentum factor.
The indicator of RBF network tuning $\mathrm{PI}^{\lambda} \mathrm{D}^{\mu}$ parameters can be calculated as follows:

$$
Z(k)=\frac{\text { error }(k)^{2}}{2}
$$

The parameters $K_{p}, K_{i}, K_{d}, \lambda$, and $\mu$ of $\mathrm{PI}^{\lambda} \mathrm{D}^{\mu}$ controller are updated through the following equations:

$$
\begin{aligned}
\Delta K_{p} & =-\eta \frac{\partial Z}{\partial K_{p}}=-\eta \frac{\partial Z}{\partial y_{m}} \frac{\partial y_{m}}{\partial u_{\mathrm{PID}}} \frac{\partial u_{\mathrm{PID}}}{\partial K_{p}} \\
& =\eta \operatorname{error}(k)^{2} \frac{\partial y_{m}}{\partial u_{\mathrm{PID}}}, \\
\Delta K_{i} & =-\eta \frac{\partial Z}{\partial K_{i}}=-\eta \frac{\partial Z}{\partial y_{m}} \frac{\partial y_{m}}{\partial u_{\mathrm{PID}}} \frac{\partial u_{\mathrm{PID}}}{\partial K_{i}} \\
& =\eta \operatorname{error}(k) \frac{\partial y_{m}}{\partial u_{\mathrm{PID}}} T^{\lambda} f_{1}, \\
\Delta K_{d} & =-\eta \frac{\partial Z}{\partial K_{d}}=-\eta \frac{\partial Z}{\partial y_{m}} \frac{\partial y_{m}}{\partial u_{\mathrm{PID}}} \frac{\partial u_{\mathrm{PID}}}{\partial K_{d}} \\
& =\eta \operatorname{error}(k) \frac{\partial y_{m}}{\partial u_{\mathrm{PID}}} T^{-\mu} f_{2}, \\
\Delta \lambda & =-\eta \frac{\partial Z}{\partial \lambda}=-\eta \frac{\partial Z}{\partial y_{m}} \frac{\partial y_{m}}{\partial u_{\mathrm{PID}}} \frac{\partial u_{\mathrm{PID}}}{\partial \lambda} \\
& =\eta \operatorname{error}(k) \frac{\partial y_{m}}{\partial u_{\mathrm{PID}}} T^{-\mu} K_{d}\left(-f_{2} \ln T+\frac{\mathrm{d} f_{2}}{\mathrm{~d} \mu}\right) \\
& =\eta \operatorname{error}(k) \frac{\partial y_{m}}{\partial u_{\mathrm{PID}}} T^{\lambda} K_{i}\left(f_{2} \ln T+\frac{\partial Z}{\partial \mu}=-\eta \frac{\partial Z}{\partial y_{m}} \frac{\partial y_{m}}{\partial u_{\mathrm{PID}}} \frac{\partial u_{\mathrm{PID}}}{\partial \mu}\right. \\
& =-\eta
\end{aligned}
$$

where $f_{1}=\sum_{i=0}^{k} q_{i} \operatorname{error}(k-i), f_{2}=\sum_{i=0}^{k} d_{i} \operatorname{error}(k-i)$ and $\partial y_{m} / \partial u_{\text {PID }}$ is the Jacobian information of the controlled object and can be acquired from the RBF identification, expressed as follows:

$$
\frac{\partial y_{m}(k)}{\partial u_{\mathrm{PID}}(k)}=\sum_{j=1}^{m} \omega_{j} h_{j} \frac{c_{j i}-u_{\mathrm{PID}}(k)}{b_{j}^{2}} .
$$

4.3. Control Algorithm. The control algorithm based on CMAC and $\mathrm{PI}^{\lambda} \mathrm{D}^{\mu}$ controller can achieve the feedforward and feedback control to improve the stability of system and give an excellent advantage of disturbance attenuation. The structure and principle of the compound control method are schematized in Figure 5, where $r_{\text {in }}$ is the expected input, $y_{\text {out }}$ is the actual output, error is the control error, $u_{c}$ is the CMAC output, $u_{\mathrm{PID}}$ is the $\mathrm{PI}^{\lambda} \mathrm{D}^{\mu}$ controller output, $u$ is the total input of VCAHC, and RBF network is applied to tuning parameters $\left(K_{p}, K_{i}, K_{d}, \mu\right.$, and $\left.\lambda\right)$ of $\mathrm{PI}^{\lambda} \mathrm{D}^{\mu}$ controller, as described in Section 4.2. The input $r_{\text {in }}$ is inputted into $\mathrm{CMAC}$ as training signals for the adjustment of weights. The CMAC and $\mathrm{PI}^{\lambda} \mathrm{D}^{\mu}$ controller contains not only the inverse 


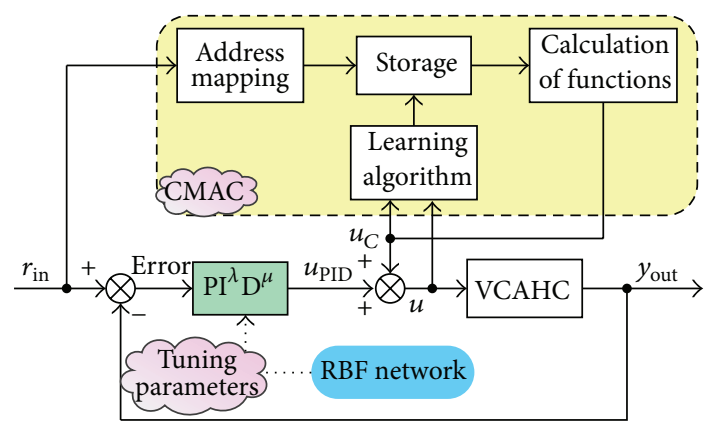

FIGURE 5: Structure and principle of the compound control based on CMAC and $\mathrm{PI}^{\lambda} \mathrm{D}^{\mu}$.

model of VCAHC, but also the inverse model of the whole system. Thus, compound control-based CMAC and $\mathrm{PI}^{\lambda} \mathrm{D}^{\mu}$ controller is helpful in overcoming the asymmetry of the VCAHC model and improving the asymmetry and tracking accuracy in movement.

As Figure 5 shows, a kind of compound control is generated through configuring CMAC in parallel with $\mathrm{PI}^{\lambda} \mathrm{D}^{\mu}$ controller. The $\mathrm{PI}^{\lambda} \mathrm{D}^{\mu}$ controller provides learning samples to the CMAC controller and plays subsidiary control function. With the system input $r_{\text {in }}$, the CMAC controller can adjust the weights dynamically using a learning algorithm. The working procedure of CMAC, including learning and control, can be described as follows.

(1) When system starts to run, the weight $w_{i}$ is set to zero. Therefore, $u_{c}$ is zero, and CMAC does not provide any control function. The VCAHC input $u=u_{\text {PID }}$ and the system are controlled by $\mathrm{PI}^{\lambda} \mathrm{D}^{\mu}$ controller.

(2) System input $r_{\text {in }}$ is inputted into CMAC after quantization. The input space $S$ is divided into $2 c+N$ quantitative intervals in $\left[S_{\min }, S_{\max }\right]$ and can be calculated by the following equations:

$$
\begin{aligned}
\Delta v & =\frac{\left(S_{\max }-S_{\min }\right)}{(N-1)}, \\
v_{i} & =S_{\min } \quad(i=1,2, \ldots, c), \\
v_{i} & =v_{i-1}+\Delta v \quad(i=c+1, \ldots, c+N), \\
v_{i} & =S_{\max } \quad(i=c+N+1, \ldots, 2 c+N) .
\end{aligned}
$$

The corresponding actual storage space can be generated by the following equation:

$$
a_{i}= \begin{cases}1 & S_{i} \in\left[v_{i}, v_{i+c}\right], i=1, \ldots, c+N \\ 0 & \text { for other } S_{i}\end{cases}
$$

where $S_{\max }$ and $S_{\min }$ are the maximum and minimum values of input $r_{\text {in }}, v_{i}$ is the quantitative value, $N$ is the number of quantitative space dimensions, $c$ is the generalization parameters of CMAC, $\mathbf{a}$ is the binary selection vector, and $\mathbf{a}=\left[a_{1}, a_{2}, \ldots, a_{c+N}\right]$.

(3) The CMAC output $u_{c}$ is calculated by adding the weight vector $\mathbf{w}$ stored in the $W$ elements and the final
VCAHC input can be obtained by adding the outputs of CMAC and $\mathrm{PI}^{\lambda} \mathrm{D}^{\mu}$ controllers, expressed as follows:

$$
\begin{aligned}
& u_{c}(k)=\mathbf{w a}^{\mathrm{T}}, \\
& u(k)=u_{\mathrm{PID}}(k)+u_{c}(k) .
\end{aligned}
$$

In every control cycle, the weights are adjusted by comparing $u_{c}$ with $u$ and this adjustment is called the learning process. The purpose of this process is to make the difference between them as small as possible. Then, after a number of learning cycles, the VCAHC input is generated mainly by the CMAC controller. The learning algorithm can be described by (17), expressed as follows:

$$
\begin{gathered}
\Delta \mathbf{w}(k)=\eta_{c}\left(1-\mathrm{e}^{-\left(u(k)-u_{c}(k)\right)}\right) \frac{u(k)-u_{c}(k)}{c} \\
\mathbf{w}(k)=\mathbf{w}(k-1)+\Delta \mathbf{w}(k) \\
+\alpha_{c}(\mathbf{w}(k)-\mathbf{w}(k-1)) .
\end{gathered}
$$

In (17), $\eta_{c}\left(1-\mathrm{e}^{-\left(u(k)-u_{c}(k)\right)}\right)$ is the learning rate of the network, where $\eta_{c}$ is a small constant and $\eta_{c} \in(0,1)$. As the reduction of $u(k)-u_{c}(k)$, the learning rate can decay by exponent regularity. When $u(k)-u_{c}(k)$ is becoming smaller, a lower learning rate will be generated and can reduce vibration and increase stability, whereas a bigger learning rate will be obtained and can accelerate the convergence procedure, $\alpha_{c}$ is the momentum factor, and $\alpha_{c} \in(0,1)$.

\section{Laboratory Experiments}

In order to verify the feasibility and effectiveness of the CMAC-PI ${ }^{\lambda} \mathrm{D}^{\mu}$ controller for hydraulically driven shearer drum lifting based on VCAHC, some experiments under different operating conditions were carried out. As the variability and randomness of coal seam distribution, the height of shearer drum should have rapid reaction speed and better tracking effect to adapt to the changes of coal seam. Therefore, two experiments of response performance analysis and tracking performance analysis were provided. Figure 6 illustrated the testing principle of the experimental system and a photograph of the experimental equipment was provided in Figure 7.

The experimental system was mainly composed of a VCAHC, a hydraulic power system, a shearer model, an external loading device, and other components. In the hydraulic power system, the motor power was $0.75 \mathrm{~kW}$ and the rated flow of pump was $6 \mathrm{~L} / \mathrm{min}$, which provided the oil pressure of $5 \mathrm{MPa}$. The stroke of the cylinder was $0.2 \mathrm{~m}$, the diameter of the piston was $0.03 \mathrm{~m}$, and the diameter of the pole was $0.018 \mathrm{~m}$. A YC-TK150S-H angle sensor was installed on the swing arm to measure the output of the VCAHC according to a mathematical relationship between the angle of swing-arm and the displacement of the pole. The external load was changed by placing different mass blocks. The controller computer was a Programmable Logic Controller (PLC) of S7-1200 with a 1214C AC/DC/Rly CPU, a CM 1242-5 ProfiBus DP-Slave module, 16 bit A/D, and 16 


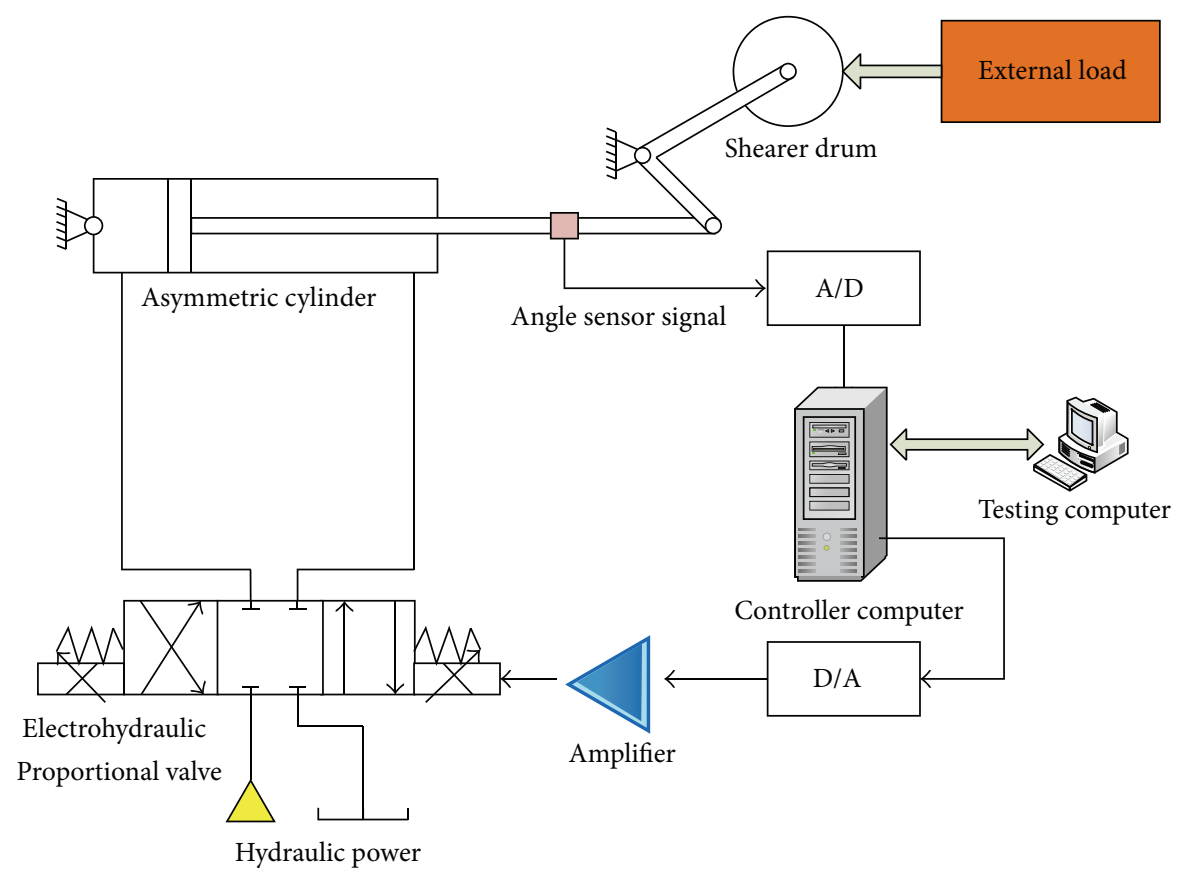

FIGURE 6: Testing principle of the experimental system.

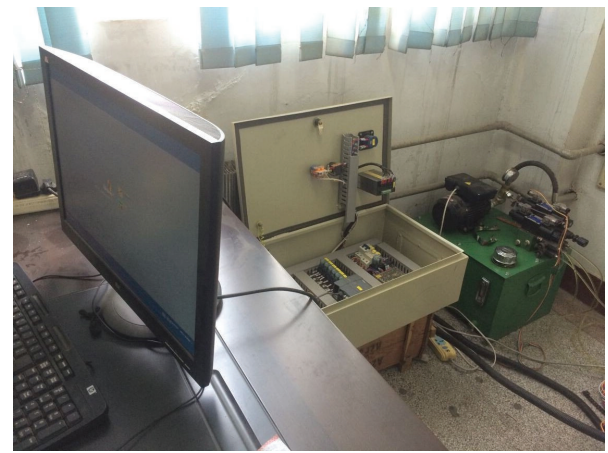

FIGURE 7: The experimental equipment.

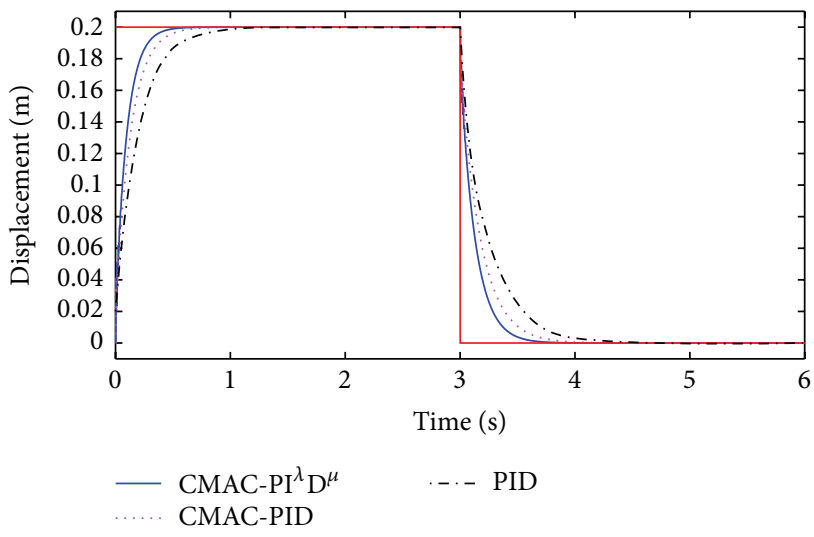

Figure 8: Comparison of displacement responses.

bit D/A. A testing computer was connected with the control computer by Ethernet and TCP/IP and was used to set the regularity of movement, sent control commands, and display the experimental data.

5.1. Experiment of Response Characteristics. To show the response performance of hydraulically driven shearer drum lifting through CMAC-PI ${ }^{\lambda} \mathrm{D}^{\mu}$ controller, comparisons with other control schemes were provided. Experiment was carried out with a single square function of $0.2 \mathrm{~m}$. Two types of motion of the asymmetric cylinder were examined: positive and negative movement. The motion of cylinder changed from forward to backward at $3 \mathrm{~s}$. The CMAC-PI ${ }^{\lambda} \mathrm{D}^{\mu}$ controller, CMAC-PID controller, and traditional PID controller were simulated under the same parameters to compare the effects of different control strategies. Figure 8 plotted the position responses of hydraulic piston under fixed loading of $50 \mathrm{~kg}$.

From Figure 8 , it could be seen that $\mathrm{CMAC}-\mathrm{PI}^{\lambda} \mathrm{D}^{\mu}$ achieved significantly good response performance on the height control of shearer drum. Four criteria, including rise time (the average rise time of positive and negative movement), settling time (the average settling time of positive and negative movement), integral absolute error (IAE), and integral of time multiplied absolute error (ITAE), were provided to show different aspects on performance of controllers. In Table 1, comparisons of used controllers in terms of rise time, settling time, and IAE and ITAE could be seen. CMAC-PI ${ }^{\lambda} \mathrm{D}^{\mu}$ controller has smaller rise time, settling time, and better IAE and ITAE values, and the performances of CMAC-PI ${ }^{\lambda} \mathrm{D}^{\mu}$ and CMAC-PID are better than PID controller. Compared 


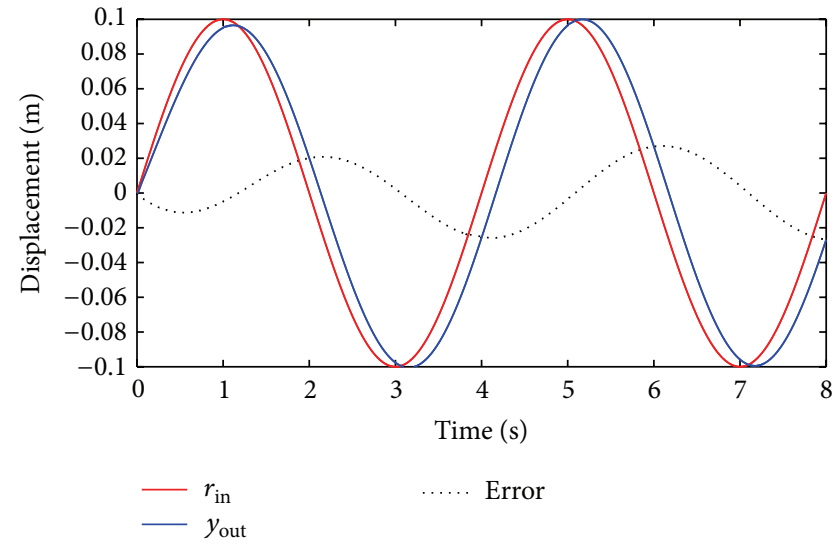

(a)

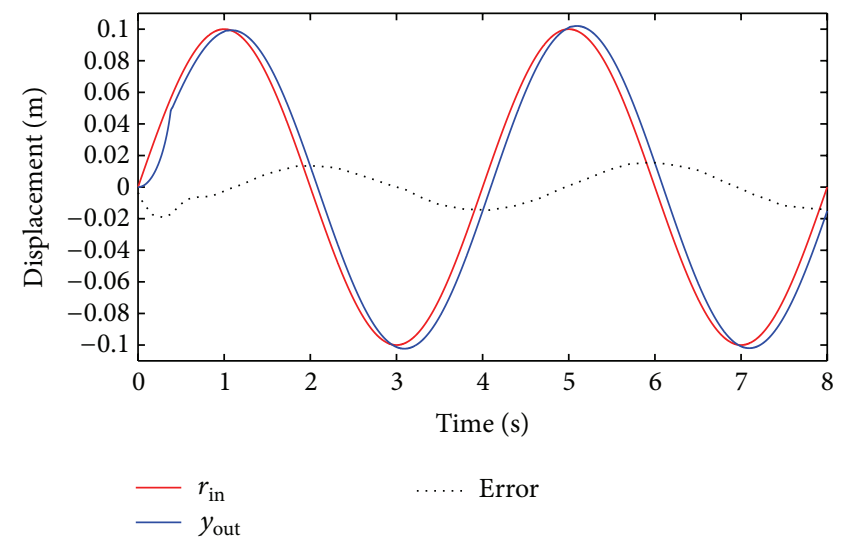

(b)

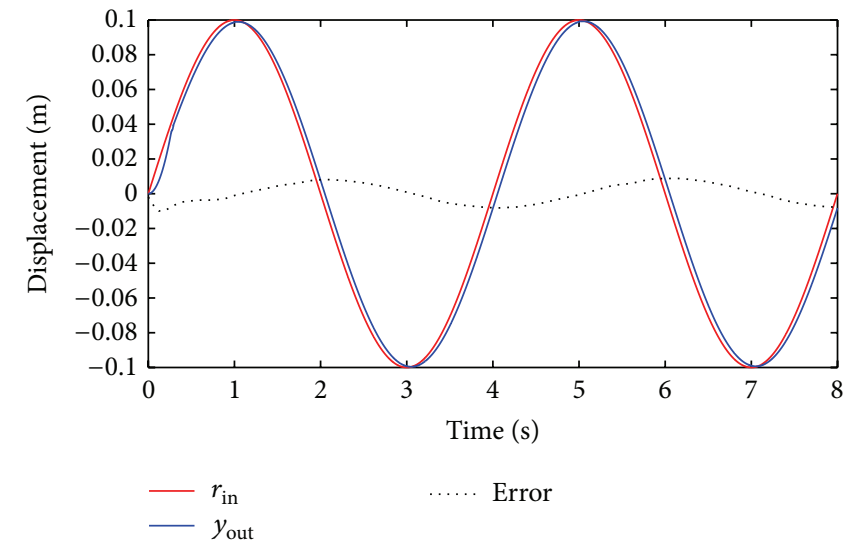

(c)

FIGURE 9: Experimental results for VCAHC of sine signal under fixed load: (a) PID controller, (b) CMAC-PID controller, and (c) CMAC$\mathrm{PI}^{\lambda} \mathrm{D}^{\mu}$ controller.

TABLE 1: Comparisons of controllers.

\begin{tabular}{lccc}
\hline Performance & PID & CMAC-PID & CMAC-PI $^{\lambda} \mathrm{D}^{\mu}$ \\
\hline Rise time (s) & 0.4524 & 0.2859 & 0.2264 \\
Settling time (s) & 0.5636 & 0.3918 & 0.3036 \\
IAE & 0.0822 & 0.0596 & 0.0416 \\
ITAE & 0.1481 & 0.1039 & 0.0704
\end{tabular}

with CMAC-PID, CMAC-PI ${ }^{\lambda} \mathrm{D}^{\mu}$ controller reduces rise time $20.8 \%$ and has $22.5 \%$ better settling time. Furthermore, CMAC-PI ${ }^{\lambda} \mathrm{D}^{\mu}$ control scheme provides smaller IAE and ITAE values than CMAC-PID does.

5.2. Experiment of Tracking Characteristics. In this experiment, the hydraulically driven shearer drum lifting-based VCAHC was tested to analyze its tracking performance under different control signals. The external load was from $50 \mathrm{~kg}$ to $120 \mathrm{~kg}$. The movement regularity of the cylinder was a sine signal, which could be expressed as follows:

$$
r_{\text {in }}=0.1 \cdot \sin (0.5 \pi t) .
$$

The CMAC-PI ${ }^{\lambda} \mathrm{D}^{\mu}$ controller, CMAC-PID controller, and traditional PID controller were tested under the same parameters to compare the effects of different control methods. Figure 9 plotted the experimental results under fixed loading of $50 \mathrm{~kg}$. As Figure 9(a) shows, the result of traditional PID controller is not ideal. The average tracking error reaches about $0.013 \mathrm{~m}$, and the degree of asymmetry, $\bar{\omega}$, as calculated by (3), is more than $46 \%$. As shown in Figures 9(b) and 9(c), the control effects of CMAC-PID controller and CMAC$\mathrm{PI}^{\lambda} \mathrm{D}^{\mu}$ controller are not better than that of PID controller at the initial stage. This is because the weights in CMAC are zero and, consequently, their adjustment requires a learning process. After about 350 control cycles, however, the compound controllers achieve ideal control precision. Compared with CMAC-PID controller, CMAC-PI ${ }^{\lambda} \mathrm{D}^{\mu}$ controller controls the tracking error of $0.004 \mathrm{~m}$ and $18 \%$ degree of asymmetry, which is less, $51 \%$ and $38 \%$, than that of CMACPID controller, respectively.

Generally, as the change of mechanical condition in coal mining, control precision was often affected by severe changing load; thus, the control effects of CMAC-PI ${ }^{\lambda} \mathrm{D}^{\mu}$ controller, CMAC-PID controller, and traditional PID controller for VCAHC under variable load should also be tested. Figure 10 

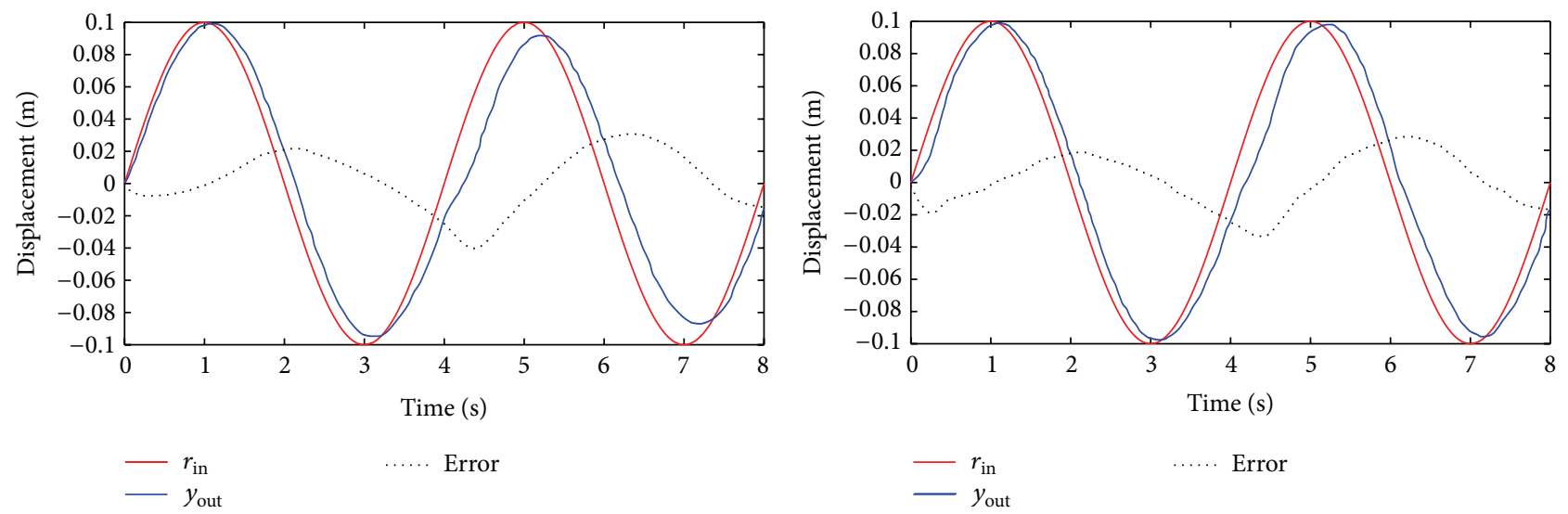

(a)

(b)

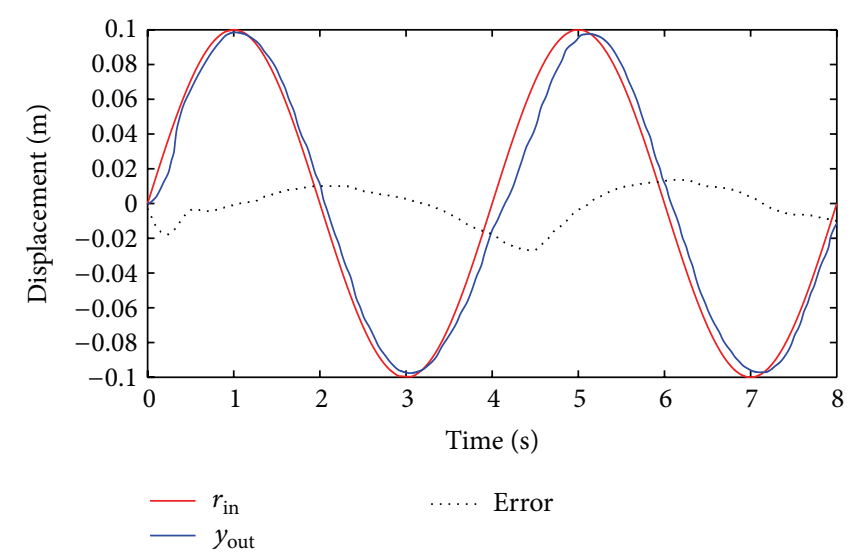

(c)

FIGURE 10: Experimental results for VCAHC of sine signal under variable load: (a) PID controller, (b) CMAC-PID controller, and (c) CMAC$\mathrm{PI}^{\lambda} \mathrm{D}^{\mu}$ controller.

plotted the results for three controllers under variable load, which changed from $70 \mathrm{~kg}$ to $120 \mathrm{~kg}$ at time of $4 \mathrm{~s}$. The control precision and the symmetry of traditional PID controller are very sensitive to variable load (Figure 10(a)). In comparison, the results of CMAC-PI ${ }^{\lambda} \mathrm{D}^{\mu}$ and CMAC-PID controllers are more ideal. The control error under variable load is reduced rapidly by CMAC-PI ${ }^{\lambda} \mathrm{D}^{\mu}$ controller and CMAC-PID controller after fast study, and the precision and symmetry can keep stable even under variable load (Figures 10(b) and 10(c)). Furthermore, compared with CMAC-PID controller, CMAC-PI ${ }^{\lambda} \mathrm{D}^{\mu}$ controller has less tracking error and better symmetry, obviously. Through the integration of CMAC and $\mathrm{PI}^{\lambda} \mathrm{D}^{\mu}$, the performance of the compound controllers was improved and the parameters were tuned by $\mathrm{RBF}$ neural network to better adapt to the mutation of external load.

From above analysis, the proposed control strategy owns perfect control performance under fixed load and variable load. Actually, as the complexity of coal seam, the stress upon drum often changes randomly. Thus, in order to reflect this variability and randomness, the movement regularity of the cylinder should be experimented with a random signal, which could be expressed as follows:

$$
\begin{aligned}
r_{\text {in }}= & 0.05 \cdot \sin (0.2 \pi t)+0.02 \cdot \sin (0.4 \pi t) \\
& +0.045 \cdot \sin (0.5 \pi t)
\end{aligned}
$$

Figure 11 plotted the results for three controllers under a fixed load of $120 \mathrm{~kg}$ and Figure 12 plotted the results under variable load, which also changed from $70 \mathrm{~kg}$ to $120 \mathrm{~kg}$ at time of $4 \mathrm{~s}$. For the random signal, the proposed control strategy could perform perfect tracking effect to adapt to the changes of coal seam. Seen from Figures 11 and 12, the control performance of proposed controller is obviously outperforming others under variable load, although its traction effect does not bear remarkable advantage than PID controller and CMAC-PID controller under fixed load. Taken Figure 12 to analyze, the control error of CMAC-PI ${ }^{\lambda} \mathrm{D}^{\mu}$ controller is about $7 \mathrm{~mm}$, which is less, $48 \%$ and $65 \%$, than that of CMACPID controller and PID controller, respectively. Although the random signal is not more regular than the sine signal, the proposed controller can also present acceptable tracking effect. 


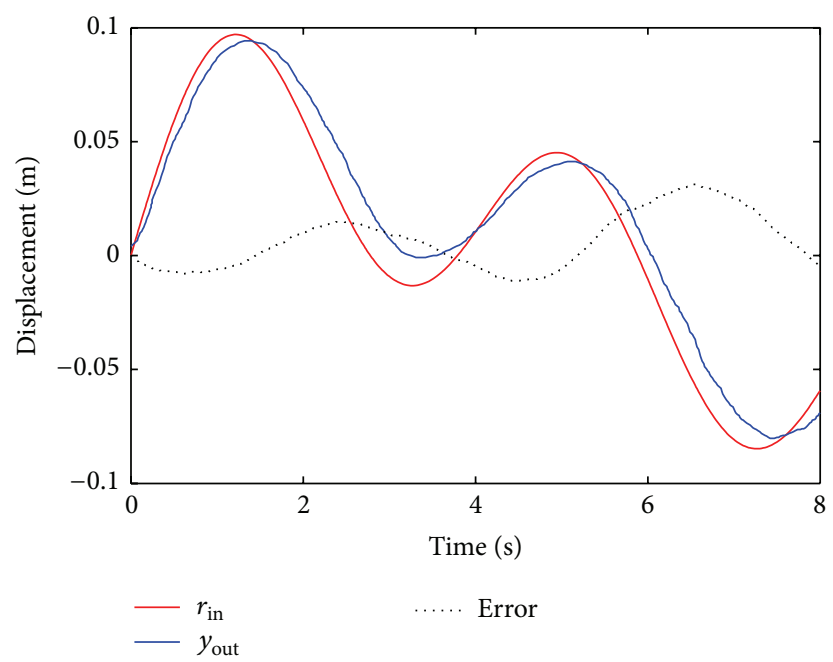

(a)

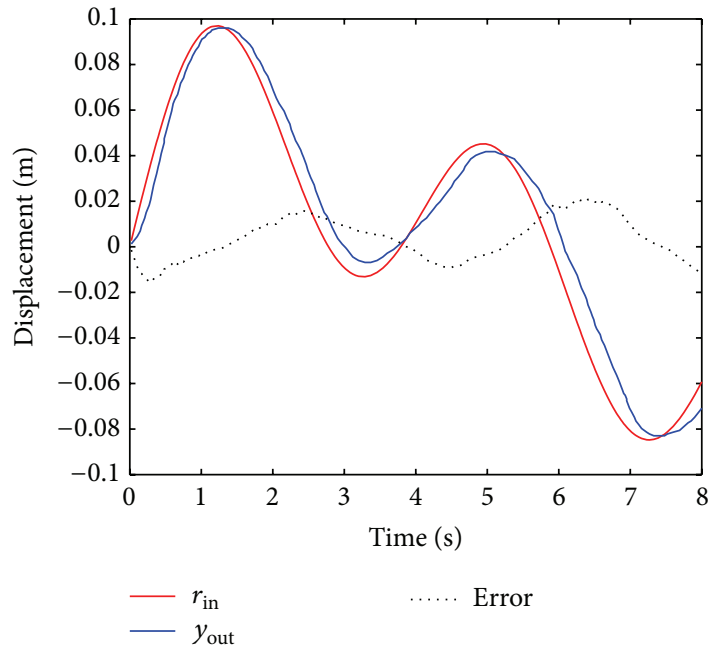

(b)

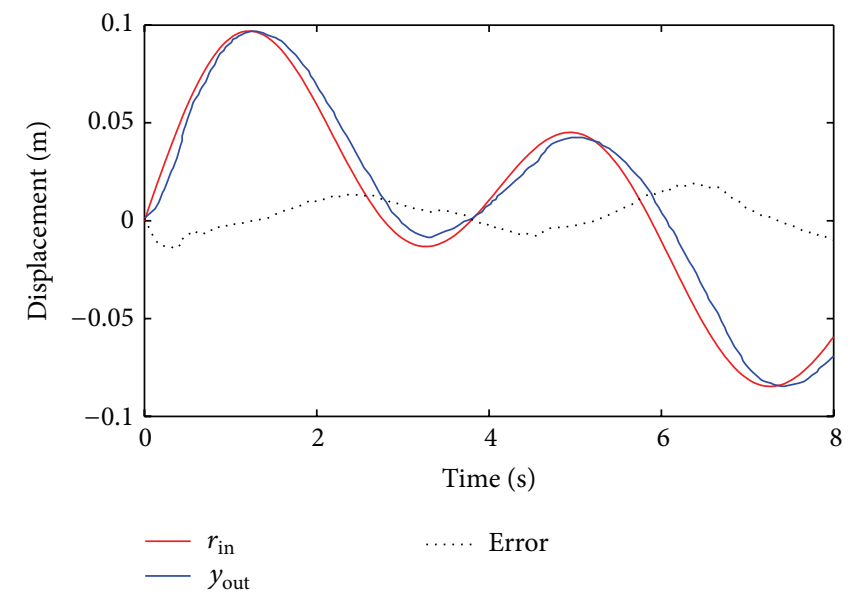

(c)

FIGURE 11: Experimental results for VCAHC of random signal under fixed load: (a) PID controller, (b) CMAC-PID controller, and (c) CMAC$\mathrm{PI}^{\lambda} \mathrm{D}^{\mu}$ controller.

From the analysis of two experiments, the controller based on proposed method can obtain better response performance and tracking effect for different control signals under variable load. The tested results indicate that the CMAC$\mathrm{PI}^{\lambda} \mathrm{D}^{\mu}$ controller is feasible and outperforming others and can be applied in the control of lifting shearer drum to better adapt to the changes of coal seam.

\section{Conclusions}

In the system of hydraulically driven shearer drum lifting, the movement precision and response speed are determined mainly by the control precision of VCAHC. Due to the asymmetrical structure of the VCAHC, it is very difficult to achieve ideal control effect with traditional PID control. Therefore, a new compound control method based on CMAC and fractional order PID controller has been proposed. Two experiments are presented to verify the validity and effectiveness of proposed control method. The theoretical analysis and testing results showed that the proposed CMAC-PI ${ }^{\lambda} \mathrm{D}^{\mu}$ controller, compared with other controllers, can achieve high movement precision and response speed and is feasible to lift shearer drum to adapt to the changes of coal seam.

\section{Conflict of Interests}

The authors declare that there is no conflict of interests regarding the publication of this paper.

\section{Acknowledgments}

The supports of the National High Technology Research and Development Program of China (no. 2013AA06A411), the National Key Basic Research Program of China: Key Fundamental Research on the Unmanned Mining Equipment in Deep Dangerous Coal Bed (no. 2014CB046300), and the Priority Academic Program Development of Jiangsu Higher 


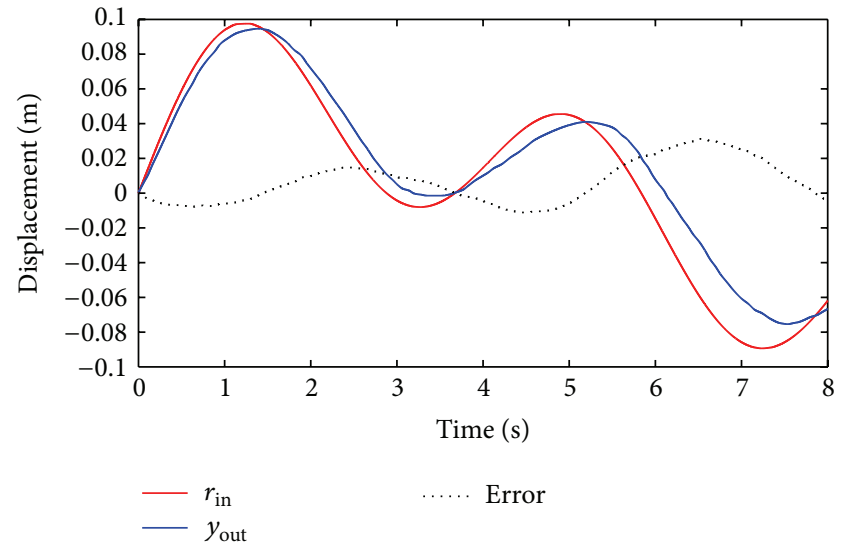

(a)

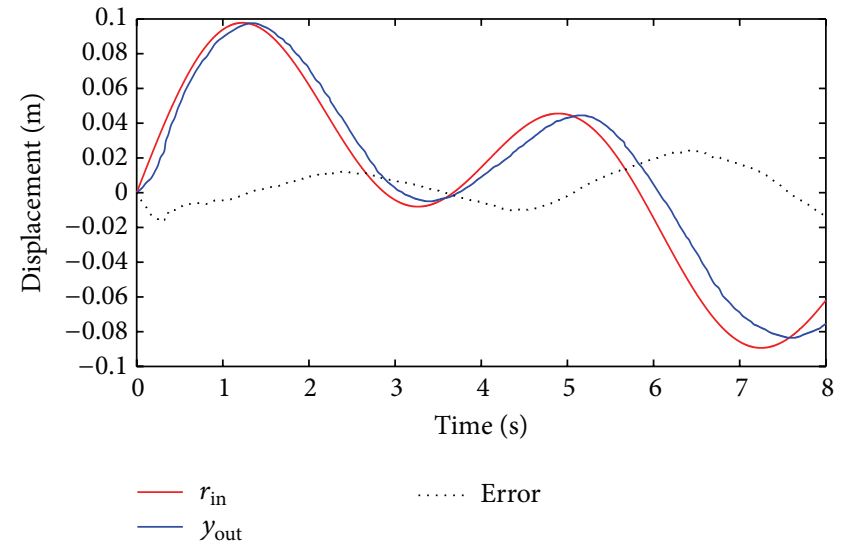

(b)

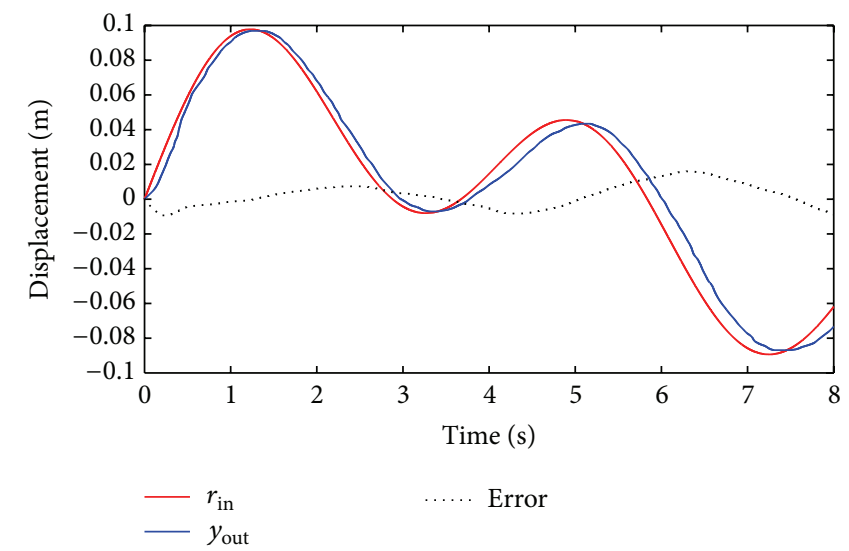

(c)

FIGURE 12: Experimental results for VCAHC of random signal under variable load: (a) PID controller, (b) CMAC-PID controller, and (c) CMAC-PI ${ }^{\lambda} \mathrm{D}^{\mu}$ controller.

Education Institutions in carrying out this research are gratefully acknowledged.

\section{References}

[1] Z. Wang, L. Si, C. Tan, and X. Liu, "A novel approach for shearer cutting load identification through integration of improved particle swarm optimization and wavelet neural network," Advances in Mechanical Engineering, vol. 2014, Article ID 521629, 13 pages, 2014.

[2] C. S. Liu, K. Jing, and Q. Yang, "A new grey correlation degree computation method of shearer drum by cutting memory program controlling," Journal of the China Coal Society, vol. 31, no. 5, pp. 666-669, 2006.

[3] C.-S. Liu, K. Jing, and Q. Yang, "Control strategy of sample intervals of cutting memory program controlling for shearer drum," Journal of Liaoning Technical University, vol. 26, no. 5, pp. 751-753, 2007.

[4] Z.-B. Wang, Z.-P. Xu, and X.-J. Dong, "Self-adaptive adjustment height of the drum in the shearer based on artificial immune and memory cutting," Journal of the China Coal Society, vol. 34, no. 10, pp. 1405-1409, 2009.
[5] Q. G. Fan, W. Li, Y. Q. Wang, M. B. Fan, and X.-F. Yang, "An algorithm of shearer memory cutting based on greyMarkovian model," Journal of Central South University (Science and Technology), vol. 42, no. 10, pp. 3054-3058, 2011.

[6] S.-H. Zhang, "Study on the innovation of fully mechanized coal shearer technology in China," Journal of the China Coal Society, vol. 35, no. 11, pp. 1898-1902, 2010.

[7] G. A. Sohl and J. E. Bobrow, "Experiments and simulations on the nonlinear control of a hydraulic servosystem," IEEE Transactions on Control Systems Technology, vol. 7, no. 2, pp. 238-247, 1999.

[8] A. V. Akkaya and Ş. Çetin, "Self tuning fuzzy logic control of a hydraulically actuating system," in Proceedings of the 2nd International Conference on Intelligent Knowledge Systems, pp. 154-158, 2005.

[9] P. E. M. Almeida and M. G. Simões, "Parametric CMAC networks: Fundamentals and applications of a fast convergence neural structure," IEEE Transactions on Industry Applications, vol. 39, no. 5, pp. 1551-1557, 2003.

[10] B. Chen and G. Liu, "Characteristic analysis of zero opening valve controlled asymmetrical cylinder system," Machine Tool and Hydraulics, vol. 36, pp. 67-70, 2008. 
[11] C.-H. Tsai and M.-F. Yeh, "Application of CMAC neural network to the control of induction motor drives," Applied Soft Computing Journal, vol. 9, no. 4, pp. 1187-1196, 2009.

[12] C.-H. Tsai, "CMAC-based speed estimator design for induction motor drive," in Proceedings of the IEEE International Conference on Systems, Man and Cybernetics, pp. 2608-2614, October 2006.

[13] X. C. Liu, J. J. Song, and T. Song, "The Study and simulation of PID control based CMAC in level control," Journal of Anshan University of Science and Technology, vol. 30, no. 4, pp. 403-406, 2007.

[14] Y. P. Wang and Y. Y. Song, "Fuzzy adaptive control based on neural network for shearer lifting system," Coal Mine Machinery, vol. 30, no. 8, pp. 188-189, 2009.

[15] G. T. Quan, C. Tan, and B. Zhou, "Study on automatic lifting hydraulic system of shearer based on fuzzy self-adaptive PID algorithm," Mining \& Processing Equipment, vol. 38, no. 15, pp. 20-23, 2010.

[16] X.-P. Su, W. Li, and Q.-G. Fan, "A shearer drum height adjusting strategy using sliding-mode variable structure control," Journal of the China Coal Society, vol. 37, no. 12, pp. 2107-2111, 2012.

[17] N.-I. Kim and C.-W. Lee, "High speed tracking control of Stewart platform manipulator via enhanced sliding mode control," in Proceedings of the IEEE International Conference on Robotics and Automation, vol. 3, pp. 2716-2721, May 1998.

[18] P. Nakkarat and S. Kuntanapreeda, "Observer-based backstepping force control of an electrohydraulic actuator," Control Engineering Practice, vol. 17, no. 8, pp. 895-902, 2009.

[19] W. M. Bessa, M. S. Dutra, and E. Kreuzer, "Sliding mode control with adaptive fuzzy dead-zone compensation of an electrohydraulic servo-system," Journal of Intelligent and Robotic Systems, vol. 58, no. 1, pp. 3-16, 2010.

[20] W. M. Bessa, "Some remarks on the boundedness and convergence properties of smooth sliding mode controllers," International Journal of Automation and Computing, vol. 6, no. 2, pp. 154-158, 2009.

[21] C.-S. Kim, K.-S. Hong, and M.-K. Kim, "Nonlinear robust control of a hydraulic elevator: experiment-based modeling and two-stage Lyapunov redesign," Control Engineering Practice, vol. 13, no. 6, pp. 789-803, 2005.

[22] J.-M. Zheng, S.-D. Zhao, and S.-G. Wei, "Fuzzy iterative learning control of electro-hydraulic servo system for SRM directdrive volume control hydraulic press," Journal of Central South University of Technology, vol. 17, no. 2, pp. 316-322, 2010.

[23] H.-M. Chen, J.-C. Renn, and J.-P. Su, "Sliding mode control with varying boundary layers for an electro-hydraulic position servo system," The International Journal of Advanced Manufacturing Technology, vol. 26, no. 1-2, pp. 117-123, 2005.

[24] J. E. D. Ekoru and J. O. Pedro, "Proportional-integral-derivative control of nonlinear half-car electro-hydraulic suspension systems," Journal of Zhejiang University: Science A, vol. 14, no. 6, pp. 401-416, 2013.

[25] D. M. Wonohadidjojo, G. Kothapalli, and M. Y. Hassan, "Position control of electro-hydraulic actuator system using fuzzy logic controller optimized by particle swarm optimization," International Journal of Automation and Computing, vol. 10, no. 3, pp. 181-193, 2013.

[26] J.-B. Lei, X.-Y. Wang, and Y.-J. Pi, "Sliding mode control in position control for asymmetrical hydraulic cylinder with chambers connected," Journal of Shanghai Jiaotong University (Science), vol. 18, no. 4, pp. 454-459, 2013.
[27] G. P. Liu and S. Daley, "Optimal-tuning nonlinear PID control of hydraulic systems," Control Engineering Practice, vol. 8, no. 9, pp. 1045-1053, 2000.

[28] C.-Y. Chen, L.-Q. Liu, C.-C. Cheng, and G. T.-C. Chiu, "Fuzzy controller design for synchronous motion in a dual-cylinder electro-hydraulic system," Control Engineering Practice, vol. 16, no. 6, pp. 658-673, 2008.

[29] L. Yuan, Y. Dong, K. Zhao, and H. Xu, "Dynamic compensator control strategy of hydraulic parallel manipulator," Journal of Beijing University of Aeronautics and Astronautics, vol. 32, no. 8, pp. 941-945, 2006.

[30] S.-K. Wang, J.-Z. Wang, and D.-W. Shi, "CMAC-based compound control of hydraulically driven 6-DOF parallel manipulator," Journal of Mechanical Science and Technology, vol. 25, no. 6, pp. 1595-1602, 2011.

[31] J. H. Yang, S. Y. Li, and Y. F. Dai, "Research on high-performance position tracking robust control strategy of a valve controlled asymmetric nonlinear system," Chinese Journal of Mechanical Engineering, vol. 18, no. 23, pp. 203-207, 2006.

[32] M. Miwa, T. Furuhashi, M. Matsuzaki, and S. Okuma, "CMAC modeling using pseudo-bacterial genetic algorithm and its acceleration," in Proceedings of the 2001 IEEE International Conference on Systems, Man and Cybernetics. e-Systems and eMan for Cybernetics in Cyberspace, pp. 250-255, October 2001. 

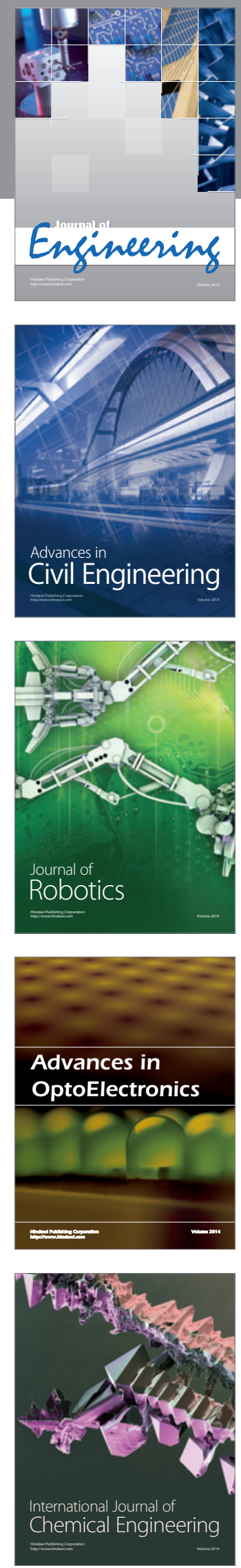

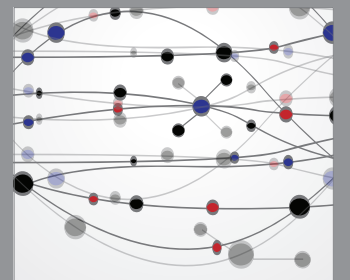

The Scientific World Journal
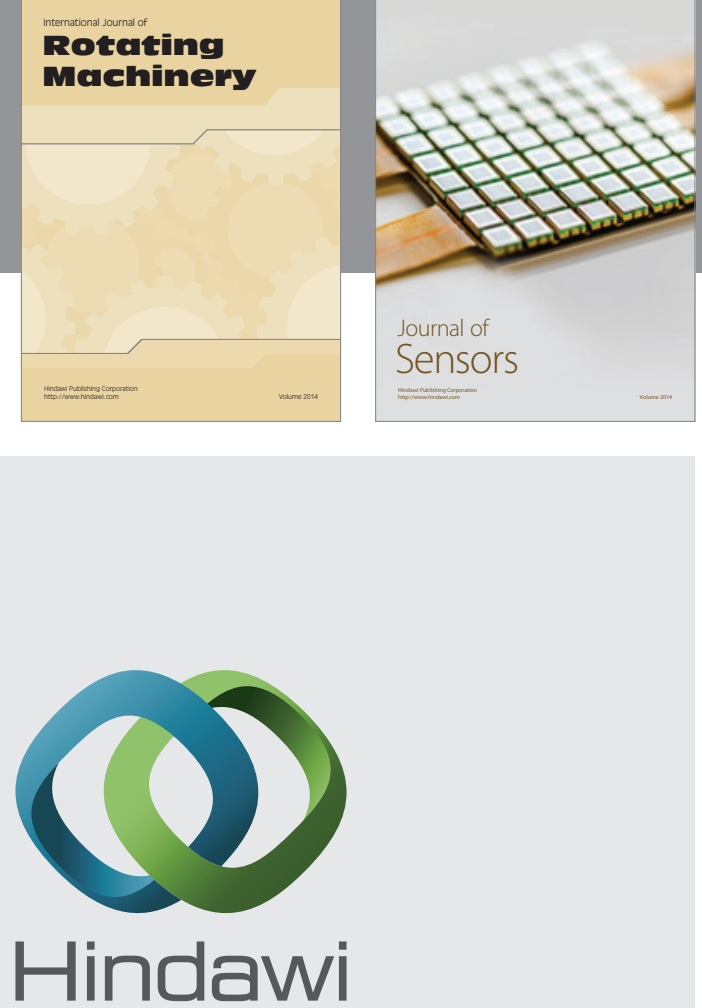

Submit your manuscripts at http://www.hindawi.com
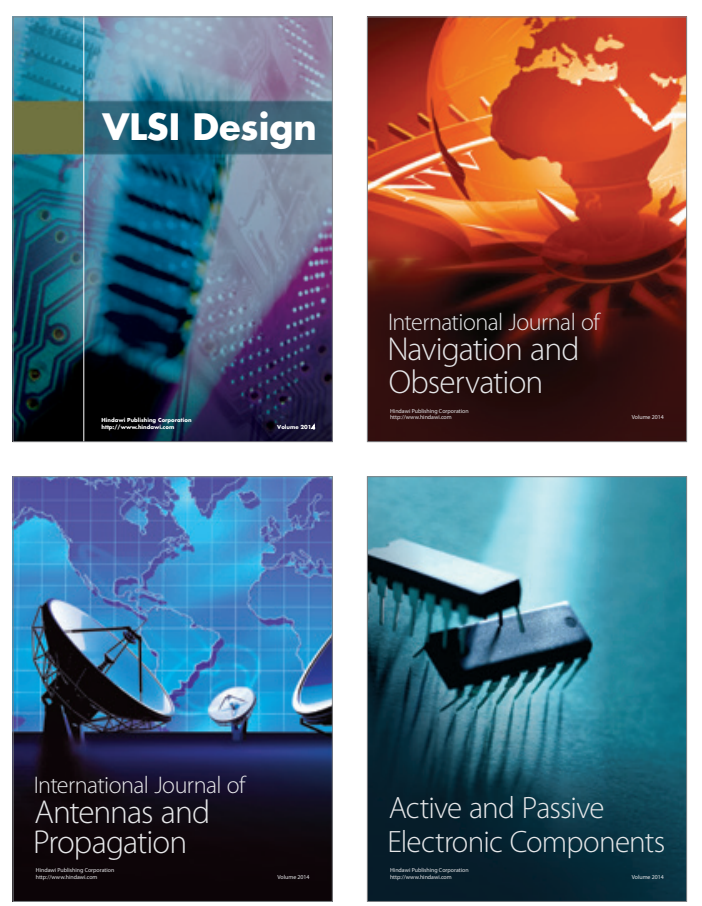
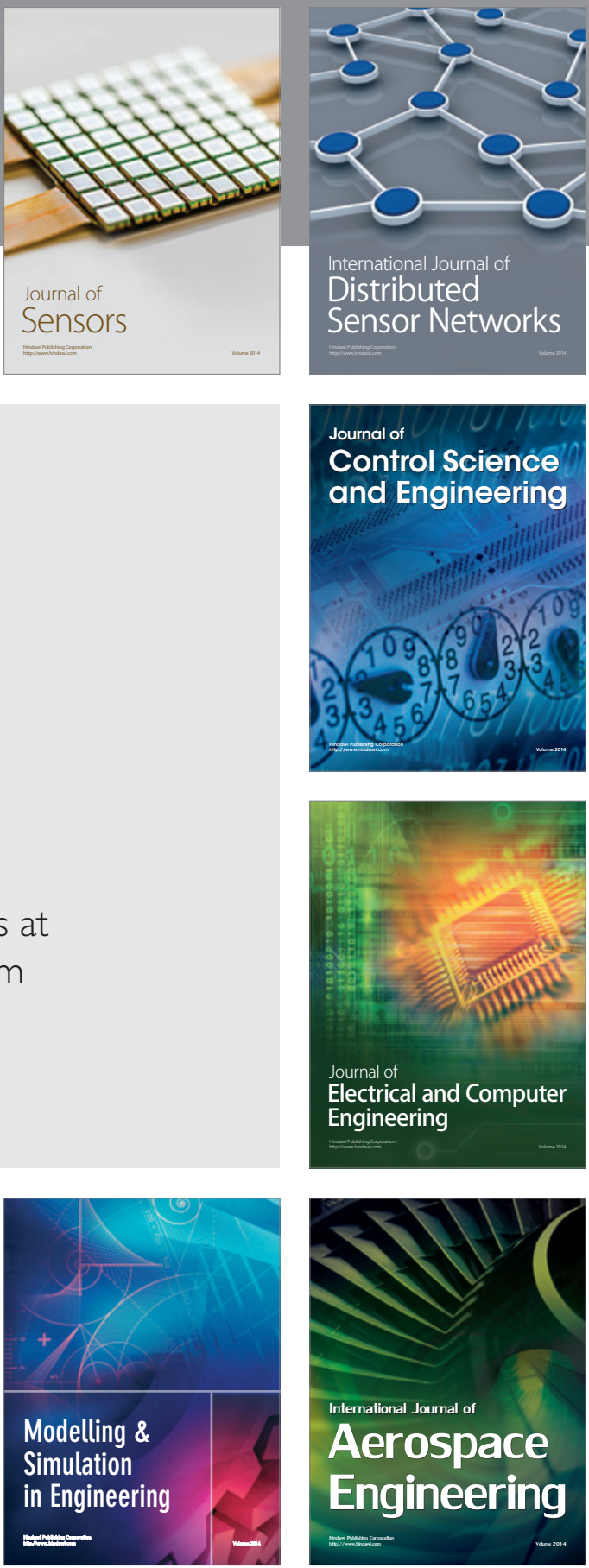

Journal of

Control Science

and Engineering
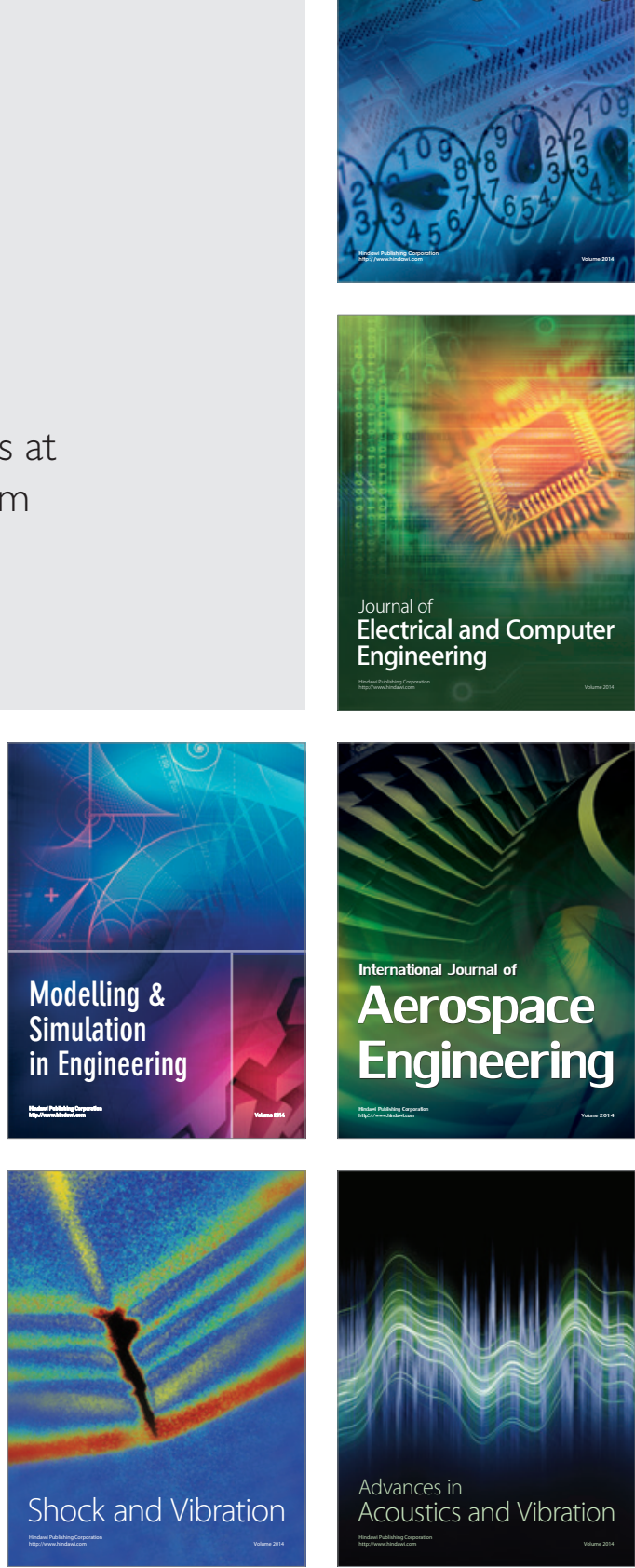